\title{
A STRATEGY FOR INVESTIGATING THE ENTEROHEPATIC CIRCULATION KINETICS OF DRUGS AND DRUG- CONJUGATE PAIRS IN INTACT ANIMALS AND HUMANS
}

\begin{abstract}
Richard H. Reuning, Yow-Ming C. Wang, Jogikal M. Jagadeesh, Ravi Subbaraya, and Valorie J. Miille.

College of Pharmacy, The Ohio State University, Columbus, $\mathrm{OH}$ 43210, USA.
\end{abstract}

The time profile for plasma concentrations of a drug, or a drug and its conjugate, may exhibit a pattern of multiple peaks and valleys when enterohepatic circulation (EHC) occurs. This happens when the drug or drug conjugate that accumulates in the gallbladder via the liver is sufficient in amount to contribute substantially to concentrations in plasma upon gallbladder emptying and subsequent reabsorption from the gastrointestinal tract. There are numerous examples of this type of behavior in the literature and the usual approach in pharmacokinetics is to ignore these peaks and valleys and obtain least squares fits to a two- or three-compartment model that does not incorporate intermittent emptying of the gallbladder.

Repetitive intermittent peaks and valleys in a plasma concentration-time curve contain valuable information about the influence of physiological processes that are not continuous, such as gallbladder emptying. The two key questions in obtaining and analyzing such information are (1) what is an appropriate pharmacokinetic model, and (2) what routes of administration and measurements of drug concentrations are needed in order to obtain valid quantitative estimates of the pharmacokinetic parameters in such a complex model? Previous attempts to analyze such data have often assumed a particular EHC model even though numerous alternative models are possible. Another inadequacy of previous studies is the lack of any analysis of the identifiability of (or theoretical ability of the available data to calculate) the pharmacokinetic parameters. Finally, the adequacy of sampling protocols is rarely analyzed.

The objective of this research is to evaluate a strategy for quantitating the pharmacokinetic parameters in complex compartmental models of EHC. We wish to achieve this objective by studying EHC as an integrated phenomenon, not as a series of separate individual steps such as biliary excretion or intestinal absorption. The four steps in our strategy are (1) establish an appropriate compartmental model by use of preliminary experiments that test various hypotheses concerning the model (2) complete an identifiability analysis for the model to determine the route(s) of administration and sampling compartment(s) that are theoretically adequate for the quantitation of all model parameters (this method assumes an infinite number of data points without error), (3) evaluate a proposed sampling protocol by carrying out computer fittings for the proposed number and timing of simulated errorfree data, with simulations obtained from estimated compartmental rate 
constants for the drug to be investigated, and (4) carry out computer fittings of data obtained by adding $10 \%$ random error to the simulated data in step 3 .

This strategy is illustrated via a four-compartment model that includes central and peripheral compartments for the drug, as well as compartments for drug in the gallbladder and intestine. Identifiability analysis of this model reveals that (1) if intravenous (iv) is the only route of administration, sampling from central, urine and gallbladder compartments is required, and (2) if administration is both iv and intraduodenal (idd), sampling from only central and urine compartments is required. Computer nonlinear least-squares fittings of simulated concentration-time data for a hypothetical EHC drug, using a rigorous sampling protocol, indicated sufficient sampling when errorfree data were used for simulations, regardless of which experimental design was used. When $10 \%$ random error was added to the simulated concentration-time data, computer fitting yielded poor estimates of known compartmental parameters with the design characterized by iv administration (only) and sampling from central, urine and gallbladder compartments. However, fits were good when administration routes were both iv and idd, whether sampling was from central and urine compartments, or from central, urine and gallbladder compartments. The latter design showed more robustness with respect to poor initial estimates for computer fitting and overlapping reabsorption and distribution exponentials.

A chronic surgery model in the rat has been developed for investigation of the strategy described above. In this model the jugular vein is cannulated for blood sampling, the proximal bile duct is cannulated for collecting bile, and the bile is returned either via a cannula inserted into the distal bile duct or via a cannula inserted directly into the duodenum. Cannulae are exteriorized for chronic sampling in a recovery surgery mode, with the bile collecting cannula connected to the bile returning cannula when sampling is completed. The two alternatives for placement of the bile-returning cannula were compared in a randomized, crossover study using rats with only jugular vein cannulation as controls. Success of the surgery was evaluated using daily measurements of body weight, bile flow rate and plasma concentrations of glutamic-pyruvic transaminase, bilirubin, lipase and creatinine. Results from all measurements indicated that returning bile via a duodenal cannulation was superior to cannulating the distal bile duct. One potential explanation for this is the disruption of branches of the pancreatic duct while cannulating the distal bile duct. This explanation is supported by much higher plasma concentrations of pancreatic lipase found in the group with the distal bile duct cannulation. The viability of the model with bile returned via the duodenal cannula was shown by the length of survival (mean $=22$ days) and the plasma concentrations of bilirubin, lipase and creatinine, which were not significantly different from controls.

A simulated gallbladder has been fabricated that is external to the rat and designed for sampling of bile and controlled first-order emptying. This engineered "gallbladder" consists primarily of a holding tank, pump, sampling 
loop, appropriate valves and a computer control system/user interface. It is connected directly to the bile-collecting and bile-returning cannulae from the surgically prepared rat. Volume in the holding tank (gallbladder equivalent) is measured via a light emitting diode array on one side and a linear photodiode array on the other. Linearity of the volume measuring system has been demonstrated. Data indicate that, under appropriate computer control, the pump empties the holding tank in a first-order manner. This simulates the physiologic behavior of gallbladder emptying.

More complex compartmental models for (1) drug-conjugate pairs, and (2) cholesterol have been analyzed using identifiability analysis. A general requirement for quantitating all models is measurement of the rate constant for emptying of the gallbladder volume. These more complex models can be solved (in theory) from measurements of only plasma concentrations if there is (1) both iv and idd administrations, (2) administration of both drug and drug conjugate, (3) quantitation of both drug and drug conjugate concentrations (or, in the case of cholesterol, both cholesterol and esterified cholesterol), and (4) inclusion of the liver within the central compartment. If the liver is modeled as a separate compartment, iv and intraportal administrations are required for quantitating the system. If only drug is administered and not the conjugate, the rate constants for the kinetic processes involving unchanged drug can be obtained, but not those for the conjugate.

Results thus far suggest that it may be possible to quantitate the individual kinetic processes of EHC in humans. However, simultaneous iv and idd administrations would likely be necessary, using different isotopic tracers, and quantitation of gallbladder emptying is an absolute requirement. The latter can be obtained via sequential ultrasound imaging of the gallbladder in humans. The potential of magnetic resonance imaging for quantitating concentrations of drugs and drug conjugates within the gallbladder in vivo offers an additional avenue of research with future promise.

The minimum requirements of (1)iv and idd administrations, (2) quantitation of drug and drug conjugate concentrations in plasma, and (3) ultrasound quantitation of the rate constant for emptying of the gallbladder offer the potential of predicting drug amounts within the gallbladder and within the intestine during the process of EHC, without actually measuring drug concentrations in these compartments in humans. This quantitation of local drug exposure may have future importance for interpreting and predicting drug responses or toxicities that are due to drug action that is localized within either of these two organs. For example, if this approach were taken with a series of new antiinfective agents, predictions of concentrations and likely effectiveness in infections of the gallbladder and biliary tree could be made without actually measuring concentrations in human bile. Then only the most promising drugs that tend to concentrate in gallbladder bile would be investigated further. 